\title{
GENERALIZED DIVERGENCE CRITERIA FOR MODEL SELECTION BETWEEN RANDOM WALK AND AR(1) MODEL
}

\section{DHAKER HAMZA ${ }^{1,2}$, PAPA NGOM ${ }^{1,2}$, PIERRE MENDY ${ }^{2}$ and EL HADJI DEME ${ }^{3}$}

\section{LMA}

Laboratoire de Mathematiques Appliquees

Senegal

e-mail: hamzaould.yahya@ucad.edu.sn

${ }^{2}$ LMDAN

Université Cheikh Anta Diop

Senegal

${ }^{3}$ LERSTAD

Université Gaston Berger

UFR SAT

Senegal

\begin{abstract}
We introduce a kernel-type estimators of $(\psi, \phi)$-divergence for continuous distributions. We discuss this approach of goodness-of-fit test for a model selection criterion relative to these divergence measures. Our interest is in the problem to testing for choosing between two models using some informational type statistics (on random walk and autoregressive AR (1)). The limit laws of the estimates and test statistics are given under both the null and the alternative hypotheses. We also describe how to apply estimators and illustrate their efficiency through numerical experiences.
\end{abstract}

2010 Mathematics Subject Classification: 94A17, 62G07, 62G10.

Keywords and phrases: divergence measure, kernel estimator, hypothesis testing.

Received May 12, 2017

(C) 2017 Scientific Advances Publishers 


\section{Introduction}

Many statistical, artificial intelligence, and machine learning problems require efficient estimation of the divergence between two distributions. We assume that these distributions are not given explicitly. Only two finite, independent and identically distributed (i.i.d.) samples are given from the two underlying distributions. Many divergence measures have been proposed since the publication of the paper of [16]. [24] gave the first generalization of Kullback-Leibler divergence, [24] defined the $J$-divergences, [4], [5], and [6] introduced the $R$-divergences, [25] the $(r, s)$-divergences, [7] the $\phi$-divergences, [27] the generalized $J$-divergences and the generalized $R$-divergences and so on. The new family of divergences, called the $(\psi, \phi)$-divergence measures, has been introduced and studied in [19].

The closest existing work most relevant to the topic of this paper is the work of Marriott and Newbold which addresses the problem of Bayesian test of the unit root problem as a Bayesian selection between two models: the random walk model and the model stationary. [17] use the same approach as [18]. This approach has been recently used in 2010 by Lynda and Hocine. In this paper, we propose another method using the divergence measures which produces very closed results to the work of Lynda and Hocine [17] and Marriot and Newbold.

The rest of this paper is organized as follows: Section 2 introduces the notation and basic definitions. Section 3 studies the asymptotic behaviour of the estimator of $(\psi, \phi)$-divergence. In Section 4 , some applications to test hypotheses are proposed. Section 5 presents some simulation results. Section 6 concludes the paper. 


\section{Formal Problem: Definitions and Estimation}

One important aspect of statistical modelling is evaluating the fit of the chosen model. Marriott and Newbold [18] discussed the Bayesian goodness of the unit root as follows:

$$
\left\{\begin{array}{l}
H_{0}: \phi=1, \\
H_{1}: \phi<1,
\end{array}\right.
$$

in the model AR(1) with intercept

$$
X_{t}-\mu 1_{d}=\phi\left(X_{t-1}-\mu 1_{d}\right)+\varepsilon_{t},
$$

where $d \in \mathbb{N}^{*}$, the $d$-dimensional vector $1_{d}=(1, \ldots, 1)^{\prime}, X_{t} \in \mathbb{R}^{d}, \forall t$ and $\varepsilon_{t}$ are i.i.d. Gaussian vector, i.e., $N\left(0_{\mathbb{R}^{d}}, \sigma^{2} \sum_{d}\right), \sum_{d}$ is the identity matrix and $\mu$ is an unknown parameter. Marriott and Newbold [18] proposed to eliminate the parameter $\mu$ considering the sample $\left(W_{1}, \ldots, W_{n}\right) \in \mathbb{R}^{d \times n}$ with zero mean vector instead of the sample $\left(X_{1}, \ldots, X_{n}\right)$ and

$$
W_{t}=X_{t}-X_{t-1}, \quad \forall t=1, \ldots, n .
$$

The authors then transformed the problem of test, a comparison between the two models, following the Bayesian approach:

$$
\begin{cases}W_{t}=\varepsilon_{t}, & (\mathrm{M} 1), \\ W_{t}-\phi W_{t-1}=\varepsilon_{t}-\varepsilon_{t-1}, & (\mathrm{M} 2) .\end{cases}
$$

Under the model (M1), the distribution function $W_{t}$ is given by:

$$
f_{1}(x)=\frac{1}{\sqrt{2 \pi \sigma^{2}}} \exp \left(-\frac{x^{\prime} \sum_{d} x}{2 \sigma^{2}}\right), \quad x \in \mathbb{R}^{d} .
$$


And under the model (M2), the distribution function $W_{t}$ can be expressed by:

$$
f_{2}(x)=\frac{1}{\sqrt{\frac{4 \pi \sigma^{2}}{1-\phi^{2}}}} \exp \left(-\frac{x^{\prime} \sum_{d} x}{\frac{4 \sigma^{2}}{1-\phi^{2}}}\right), \quad x \in \mathbb{R}^{d} .
$$

Based on their methods, we propose a new approach based on the $(\psi, \phi)$-divergence in order to find a goodness-of-fit of the model.

\subsection{A brief review of $(\psi, \phi)$-divergence}

The $\phi$-divergence measure between the probability distributions $p$ and $q$ is defined by

$$
\mathcal{D}_{\phi}(p, q)=\int_{\mathbb{R}^{d}} \phi\left(\frac{p(x)}{q(x)}\right) q(x) d x, \quad \phi \in \Phi^{*},
$$

where $\Phi^{*}$ is the class of all convex function $\phi(x), x>0$, such that at $x=1, \phi(1)=0$, at $x=0,0 \phi\left(\frac{0}{0}\right)=0$ and $0 \phi\left(\frac{p}{0}\right)=\lim _{u \rightarrow 0} \frac{\phi(u)}{u}$.

For example: $\phi(x)=x \log (x)-x+1$, we have Kullback-Leibler divergence

$$
\mathcal{D}^{K L}(p, q)=\int_{\mathbb{R}^{d}} p(x) \log \frac{p(x)}{q(x)} d x .
$$

Rényi [24] presented the first parametric generalization of KullbackLeibler

$$
\mathcal{D}_{\alpha}^{R}(p, q)=\frac{1}{\alpha-1} \log \int_{\mathbb{R}^{d}}\left(\frac{p(x)}{q(x)}\right)^{\alpha} q(x) d x
$$


It is easy to prove that

$$
\begin{aligned}
& \lim _{\alpha \rightarrow 1} \mathcal{D}_{\alpha}^{R}(p, q)=\mathcal{D}^{K L}(p, q)=\int_{\mathbb{R}^{d}} p(x) \log \frac{p(x)}{q(x)} d x, \\
& \lim _{\alpha \rightarrow 0} \mathcal{D}_{\alpha}^{R}(q, p)=\mathcal{D}^{K L}(q, p)=\int_{\mathbb{R}^{d}} q(x) \log \frac{q(x)}{p(x)} d x .
\end{aligned}
$$

Rényi are not $\phi$-divergences measures. However, such measures can be written in the following form:

$$
\mathcal{D}_{\phi}^{\psi}(p, q)=\psi\left(\mathcal{D}_{\phi}(p, q)\right)
$$

where $\psi$ is a differentiable increasing real function mapping from

$$
\left[0, \phi(0) \lim _{t \rightarrow \infty} \frac{\phi(t)}{t}\right]
$$

onto $[0, \infty)$; this condition will be justified in (Proposition 1.1, [20]), with $\psi(0)=0, \psi^{\prime}(0)>0$, and $\phi \in \Phi^{*}$. In the following formulas, we list the functions $\psi$ and $\phi$ that yield to the Rényi divergence measures:

Rényi:

$$
\psi(x)=\frac{1}{\alpha(\alpha-1)} \log (\alpha(\alpha-1) x+1), \quad \phi(x)=\frac{x^{\alpha}-\alpha(x-1)-1}{\alpha(\alpha-1)}, \alpha \neq 0,1 ;
$$

Sharma-Mittal

$\psi(x)=\frac{1}{(s-1)}\left((1+\alpha(\alpha-1) x)^{\frac{s-1}{r-1}}-1\right), \quad \phi(x)=\frac{x^{\alpha}-\alpha(x-1)-1}{\alpha(\alpha-1)}, s \neq 1, \alpha \neq 0,1 ;$

Bhattacharyya

$$
\psi(x)=-\log (-x+1), \quad \phi(x)=-x^{\frac{1}{2}}+\frac{1}{2}(x+1) .
$$

Now, let $f$ be the unknown true density function (with respect to Lebesgue measure on $\left.\mathbb{R}^{d}\right)$ of the sample $\left(W_{1}, \ldots, W_{n}\right)$ with cumulative distribution function $F$. The distance between true density and those of 
the models can be measured by the Rényi-divergence of $f$ and $f_{j}, j=1,2$ as follows:

$$
\mathcal{D}_{\phi}^{\psi}\left(f, f_{j}\right)=\psi\left(\mathcal{D}_{\phi}\left(f, f_{j}\right)\right)
$$

For a given density of probability $g$ defined on $\mathbb{R}^{d}$, we start by giving some notation and conditions that are needed for the forthcoming sections. Below, we will work under the following assumptions on $f$ and $g$ to establish our results.

(F.1) The functional $\mathcal{D}_{\phi}^{\psi}(f, g)$ as well-defined as (2), in the sense that $\mathcal{D}_{\phi}^{\psi}(f, g)$ is finite.

\subsection{Nonparametric estimation of $(\psi, \phi)$-divergence}

To define our divergence estimator we define, in a first step, a kernel density estimator. Towards this aim, we introduce a measurable function $K(\cdot)$ fulfilling the following conditions:

(K.1) $K(\cdot)$ is of bounded variation on $\mathbb{R}^{d}$,

(K.2) $K(\cdot)$ is right continuous on $\mathbb{R}^{d}$,

(K.3) $\|K\|_{\infty}=\sup _{x \in \mathbb{R}^{d}}|K(x)|<\infty$,

(K.4) $\int_{\mathbb{R}^{d}} K(t) d t=1$.

The well known Akaike-Parzen-Rosenblatt (refer to [1], [21], and [26]) kernel estimator of $f(\cdot))$ is defined, for any $x \in \mathbb{R}^{d}$, by

$$
\widehat{f}_{n, h}(x)=\frac{1}{n h^{d}} \sum_{i=1}^{n} K\left(\frac{x-W_{i}}{h}\right),
$$

where $0<h_{n} \leq 1$ is the smoothing parameter. Assuming that the density $f$ is continuous, one can obtain the normality asymptotic of the 
estimator $\widehat{f}_{n, h_{n}}$ under conditions below, see [17]. For more details of kernel estimators $\widehat{f}_{n, h_{n}}$, one can refer to [11], [12], [3], [23], [8], [14], [10] and the references therein, and their limiting behaviour.

In a second step, given $\widehat{f}_{n, h_{n}}(\cdot)$, we estimate divergence $\mathcal{D}_{\phi}^{\psi}(f, g)$ by using the representation (2) with $f$ and $g$, by setting

$$
\begin{aligned}
\widehat{\mathcal{D}}_{\phi}^{\phi}\left(\widehat{f}_{n, h_{n}}, g\right) & =\psi\left(\widehat{\mathcal{D}}_{\phi}\left(\widehat{f}_{n, h_{n}}, g\right)\right), \\
& =\psi\left(\int_{\mathbb{R}^{d}} \phi\left(\frac{\widehat{f}_{n, h_{n}}(x)}{g(x)}\right) g(x) d x\right) .
\end{aligned}
$$

The approach use to define the plug-in estimator $\widehat{\mathcal{D}}_{\phi}^{\psi}\left(\widehat{f}_{n, h_{n}}, g\right)$ is respectively developed in [2] and [15] in order to introduce a kernel-type estimators of Shannon's entropy and divergences.

In the next section, we wish to establish the asymptotic behaviour for the estimate $\widehat{\mathcal{D}}_{\phi}^{\psi}\left(\widehat{f}_{n, h_{n}}, g\right)$ and to give in application for testing hypothesis.

\section{Main Results}

We first study the asymptotic normality of the term given in the function $\psi$ and to deduce those of the general estimator.

Lemma 1. Let $K(\cdot)$ satisfy (K.1)-(K.4) and let $f(\cdot)$ be a bounded density fulfill (F.1). Suppose that $\phi \in C^{1}([0, \infty))$ and there exist a measurable and Lebesgue-integrable function $F(x)$ such that $\left|\phi^{\prime}\left(\frac{f(x)}{g(x)}\right)\right|<F(x)$. 
Then

$$
\begin{array}{r}
\sqrt{n h_{n}^{d}}\left(\int_{\mathbb{R}^{d}} \phi\left(\frac{\hat{f}_{n, h_{n}}(x)}{g(x)}\right) g(x) d x-\int_{\mathbb{R}^{d}} \phi\left(\frac{f(x)}{g(x)}\right) g(x) d x\right) \\
\left.\rightarrow \mathcal{N}\left(0,\left(\int_{\mathbb{R}^{d}} \sigma(x) \phi^{\prime}\left(\frac{f(x)}{g(x)}\right) d x\right)\right)^{2}\right),
\end{array}
$$

where $\sigma^{2}(x):=f(x) \int K^{2}(z) d z$.

Proof. The first order Taylor expansion of $\phi\left(\frac{\widehat{f}_{n, h_{n}}(x)}{g(x)}\right)$ around $\frac{f(x)}{g(x)}$ gives

$$
\begin{aligned}
& \int_{\mathbb{R}^{d}} \phi\left(\frac{\left.\hat{f}_{n, h_{n}(x)}\right) g(x)}{g(x) d x=} \int_{\mathbb{R}^{d}} \phi\left(\frac{f(x)}{g(x)}\right) g(x) d x\right. \\
&+\int_{\mathbb{R}^{d}}\left(\frac{\widehat{f}_{n, h_{n}}(x)}{g(x)}-\frac{f(x)}{g(x)}\right) \phi^{\prime}\left(\frac{f(x)}{g(x)}\right) g(x) d x \\
&+\int_{\mathbb{R}^{d}} o\left(\left\|\frac{\widehat{f}_{n, h_{n}}}{g}-\frac{f}{g}\right\|\right) g(x) d x, \\
& \int_{\mathbb{R}^{d}} \phi\left(\frac{\widehat{f}_{n, h_{n}}(x)}{g(x)}\right) g(x) d x-\int_{\mathbb{R}^{d}} \phi\left(\frac{f(x)}{g(x)}\right) g(x) d x \\
&=\int_{\mathbb{R}^{d}}\left(\widehat{f}_{n h_{n}}(x)-f(x)\right) \phi^{\prime}\left(\frac{f(x)}{g(x)}\right) d x \\
&+\int_{\mathbb{R}^{d}} o\left(\left\|\frac{\hat{f}_{n, h_{n}}}{g}-\frac{f}{g}\right\|\right) g(x) d x .
\end{aligned}
$$


Since

$$
\sqrt{n h_{n}^{d}}\left(\widehat{f}_{n h_{n}}(x)-f(x)\right) \rightarrow \mathcal{N}\left(0, \sigma^{2}(x)\right)
$$

Then $\sqrt{n h_{n}^{d}} \int_{\mathbb{R}^{d}} o\left(\left\|\frac{\widehat{f}_{n, h_{n}}}{g}-\frac{f}{g}\right\|\right) g(x) d x=\sqrt{n h_{n}^{d}} o\left(O_{p}\left(\left(n h_{n}^{d}\right)^{\frac{-1}{2}}\right)\right) \int_{\mathbb{R}^{d}} g(x) d x$ $=0_{p}(1)$.

Therefore, the random variables

$$
\left.\sqrt{n h_{n}^{d}}\left(\int_{\mathbb{R}^{d}} \phi\left(\frac{\widehat{f}_{n, h_{n}}(x)}{g(x)}\right) g(x) d x-\int_{\mathbb{R}^{d}} \phi\left(\frac{f(x)}{g(x)}\right) g(x) d x\right)\right),
$$

and

$$
\sqrt{n h_{n}^{d}} \int_{\mathbb{R}^{d}} \phi^{\prime}\left(\frac{f(x)}{g(x)}\right)\left(\widehat{f}_{n h_{n}}(x)-f(x)\right) d x
$$

have the same asymptotic distribution. By (5) we have

$$
\left.\sqrt{n h_{n}^{d}} \int_{\mathbb{R}^{d}} \phi^{\prime}\left(\frac{f(x)}{g(x)}\right)\left(\frac{\hat{f}_{n, h_{n}}(x)}{g(x)}-\frac{f(x)}{g(x)}\right) d x \rightarrow \mathcal{N}\left(0,\left(\int_{\mathbb{R}^{d}} \sigma(x) \phi^{\prime}\left(\frac{f(x)}{g(x)}\right) d x\right)\right)^{2}\right) .
$$

Theorem 1. We consider the $\mathcal{D}_{\phi}^{\psi}(f, g)$ defined in (2), then we have

$$
\begin{aligned}
& \sqrt{n h_{n}^{d}}\left(\widehat{\mathcal{D}}_{\phi}^{\psi}\left(\widehat{f}_{n, h_{n}}, g\right)-\mathcal{D}_{\phi}^{\psi}(f, g)\right) \\
& \quad \rightarrow \mathcal{N}\left(0,\left\{\psi^{\prime}\left(\int_{\mathbb{R}^{d}} \phi\left(\frac{f(x)}{g(x)}\right) g(x) d x\right) \int_{\mathbb{R}^{d}} \sigma(x) \phi^{\prime}\left(\frac{f(x)}{g(x)}\right) d x\right\}^{2}\right) .
\end{aligned}
$$

Proof. A first order Taylor expansion of $\psi(y)$ around $y=y_{0}$ at $y=\widehat{y}$ gives

$$
\psi(\widehat{y})=\psi\left(y_{0}\right)+\psi^{\prime}\left(y_{0}\right)\left(\widehat{y}-y_{0}\right)+o\left(\left\|\widehat{y}-y_{0}\right\|\right)
$$


Now for $y_{0}=\int_{\mathbb{R}^{d}} \phi\left(\frac{f(x)}{g(x)}\right) g(x) d x$ and $\hat{y}=\int_{\mathbb{R}^{d}} \phi\left(\frac{\hat{f}_{n, h_{n}}(x)}{g(x)}\right) g(x) d x$, we get

$$
\begin{aligned}
\psi\left(\int_{\mathbb{R}^{d}} \phi\left(\frac{\widehat{f}_{n, h_{n}}(x)}{g(x)}\right) g(x) d x\right)=\psi\left(\int_{\mathbb{R}^{d}} \phi\left(\frac{f(x)}{g(x)}\right) g(x) d x\right) \\
+\psi^{\prime}\left(\int_{\mathbb{R}^{d}} \phi\left(\frac{f(x)}{g(x)}\right) g(x) d x\right) \\
\times\left[\int_{\mathbb{R}^{d}} \phi\left(\frac{\widehat{f}_{n, h_{n}}(x)}{g(x)}\right) g(x) d x-\int_{\mathbb{R}^{d}} \phi\left(\frac{f(x)}{g(x)}\right) g(x) d x\right] \\
+o\left(\left\|\int_{\mathbb{R}^{d}} \phi\left(\frac{\widehat{f}_{n, h_{n}}(x)}{g(x)}\right) g(x) d x-\int_{\mathbb{R}^{d}} \phi\left(\frac{f(x)}{g(x)}\right) g(x) d x\right\|\right) \\
\widehat{\mathcal{D}}_{\phi}^{\psi}\left(\widehat{f}_{n, h_{n}}, g\right)= \\
+\mathcal{D}_{\phi}^{\psi}(f, g)+\psi^{\prime}\left(\int_{\mathbb{R}^{d}} \phi\left(\frac{f(x)}{g(x)}\right) g(x) d(x)\right) \\
+\left[\int_{\left.\mathbb{R}^{d} \phi\left(\frac{\hat{f}_{n, h_{n}}(x)}{g(x)}\right) g(x) d x-\int_{\mathbb{R}^{d}} \phi\left(\frac{f(x)}{g(x)}\right) g(x) d x\right]}\right. \\
+o\left(\left\|\int_{\mathbb{R}^{d}} \phi\left(\frac{\widehat{f}_{n, h_{n}}(x)}{g(x)}\right) g(x) d x-\int_{\mathbb{R}^{d}} \phi\left(\frac{f(x)}{g(x)}\right) g(x) d x\right\|\right)
\end{aligned}
$$

and therefore,

$$
\begin{array}{r}
\sqrt{n h_{n}^{d}}\left[\widehat{\mathcal{D}}_{\phi}^{\psi}\left(\widehat{f}_{n, h_{n}}, g\right)-\mathcal{D}_{\phi}^{\psi}(f, g)\right]=\sqrt{n h_{n}^{d}} \psi^{\prime}\left(\int_{\mathbb{R}^{d}} \phi\left(\frac{f(x)}{g(x)}\right) g(x) d x\right) \\
\times\left[\int_{\mathbb{R}^{d}} \phi\left(\frac{\widehat{f}_{n, h_{n}}(x)}{g(x)}\right) g(x) d x-\int_{\mathbb{R}^{d}} \phi\left(\frac{f(x)}{g(x)}\right) g(x) d x\right]+o_{p}(1), \\
\text { because } \sqrt{n h_{n}^{d}} o\left(\left\|\int_{\mathbb{R}^{d}} \phi\left(\frac{\widehat{f}_{n, h_{n}}(x)}{g(x)}\right) g(x) d x-\int_{\mathbb{R}^{d}} \phi\left(\frac{f(x)}{g(x)}\right) g(x) d x\right\|\right)=o_{p}(1) .
\end{array}
$$

Therefore, 


$$
\begin{aligned}
& \sqrt{n h_{n}^{d}}\left(\widehat{\mathcal{D}}_{\phi}^{\psi}\left(\widehat{f}_{n, h_{n}}, g\right)-\mathcal{D}_{\phi}^{\psi}(f, g)\right) \\
& \quad \rightarrow \mathcal{N}\left(0,\left\{\psi^{\prime}\left(\int_{\mathbb{R}^{d}} \phi\left(\frac{f(x)}{g(x)}\right) g(x) d x\right) \int_{\mathbb{R}^{d}} \sigma(x) \phi^{\prime}\left(\frac{f(x)}{g(x)}\right) d x\right\}^{2}\right)
\end{aligned}
$$

\section{Applications for Testing Hypothesis}

In this section, we use the estimators $\widehat{\mathcal{D}}_{\phi}^{\psi}\left(\widehat{f}_{n, h_{n}}, f_{j}\right) j=1,2$ to find the perform statistical tests on the model defined in Section 2.

\subsection{Goodness-of-fit test}

For completeness, we look at $\widehat{\mathcal{D}}_{\phi}^{\psi}\left(\widehat{f}_{n, h_{n}}, f_{j}\right)$ in the usual way, i.e., as a goodness-of-fit statistic. From the uniform-in-bandwidth consistency of $\widehat{\mathcal{D}}_{\phi}^{\psi}\left(\widehat{f}_{n, h_{n}}, f_{j}\right)$ for $\mathcal{D}_{\alpha}^{R}\left(f, f_{j}\right)$, the null hypothesis when using the statistic $\widehat{\mathcal{D}}_{\alpha}^{R}\left(\widehat{f}_{n, h_{n}}, f_{j}\right)$ can be given as $H_{0}: \mathcal{D}_{\phi}^{\psi}\left(f, f_{j}\right)=0$. Under the alternative hypothesis $H_{1}: \mathcal{D}_{\phi}^{\psi}\left(f, f_{j}\right) \neq 0$.

\subsection{Test for model selection}

Introduce the divergence indicator $\mathcal{D} \mathcal{I}=\mathcal{D}_{\phi}^{\psi}\left(f, f_{1}\right)-\mathcal{D}_{\phi}^{\psi}\left(f, f_{2}\right)=\mathcal{D}_{1}-\mathcal{D}_{2}$. An estimator of the divergence indicator is defined as:

$$
\widehat{\mathcal{D I}}_{n}:=\widehat{\mathcal{D}}_{\phi}^{\psi}\left(\widehat{f}_{n h_{n}}, f_{1}\right)-\widehat{\mathcal{D}}_{\phi}^{\psi}\left(\widehat{f}_{n h_{n}}, f_{2}\right)=\widehat{\mathcal{D}}_{1}-\widehat{\mathcal{D}}_{2}
$$

Using the divergence indicator, we develop the following test hypothesis on the model under study:

- $H_{0}^{e q}: \mathcal{D I}=0$ means that the two models are equivalent.

- $H_{1}^{M_{1}}: \mathcal{D I}<0$ means that model $M_{1}$ is better than model $M_{2}$.

- $H_{1}^{M_{2}}: \mathcal{D I}>0$ means that model $M_{2}$ is better than model $M_{1}$. 
$\widehat{\mathcal{D I}}_{n}$ converges to zero under the null hypothesis $H_{0}^{e q}$, but it converges to a strictly negative or positive constant when $H_{1}^{M_{1}}$ or $H_{1}^{M_{2}}$ hold. These properties actually justify the use of $\widehat{\mathcal{D I}}_{n}$ as a model selection indicator and common procedure of selecting the model with highest goodness-of-fit.

Theorem 2. Under the assumptions of Theorem 2,

(1) Under the null hypothesis $H_{0}^{e q}, \sqrt{n h_{n}^{d}} \widehat{\mathcal{D I}}_{n} \rightarrow \mathcal{N}\left(0, \Gamma^{2}\right)$;

(2) Under the $H_{1}^{M_{1}}$ hypothesis $\sqrt{n h_{n}^{d}} \widehat{\mathcal{D I}}_{n} \rightarrow-\infty$;

(3) Under the $H_{1}^{M_{2}}$ hypothesis $\sqrt{n h_{n}^{d}} \widehat{\mathcal{D I}}_{n} \rightarrow+\infty$,

with

$$
\begin{aligned}
\Gamma^{2}=\left\{\int _ { \mathbb { R } ^ { d } } \left[\psi^{\prime}\left(\int_{\mathbb{R}^{d}} \phi\left(\frac{f(x)}{f_{1}(x)}\right) f_{1}(x) d x\right) \phi^{\prime}\left(\frac{f(x)}{f_{1}(x)}\right)\right.\right. \\
\left.\left.\quad-\psi^{\prime}\left(\int_{\mathbb{R}^{d}} \phi\left(\frac{f(x)}{f_{2}(x)}\right) f_{2}(x) d x\right) \phi^{\prime}\left(\frac{f(x)}{f_{2}(x)}\right)\right] \sigma(x) d x\right\}^{2} .
\end{aligned}
$$

Proof.

$$
\begin{aligned}
\widehat{\mathcal{D I}}_{n}: & =\widehat{\mathcal{D}}_{\phi}^{\varphi}\left(\widehat{f}_{n, h_{n}}, f_{1}\right)-\widehat{\mathcal{D}}_{\phi}^{\varphi}\left(\widehat{f}_{n, h_{n}}, f_{2}\right) \\
& =\sqrt{n h_{n}^{d}}\left\{\left[\widehat{\mathcal{D}}_{1}-\mathcal{D}_{1}\right]-\left[\widehat{\mathcal{D}}_{2}-\mathcal{D}_{2}\right]\right\}+\sqrt{n h_{n}^{d}}\left\{\left[\mathcal{D}_{1}-\mathcal{D}_{2}\right]\right\} \\
& =\sqrt{n h_{n}^{d}}\left\{\left[\widehat{\mathcal{D}}_{1}-\mathcal{D}_{1}\right]-\left[\widehat{\mathcal{D}}_{2}-\mathcal{D}_{2}\right]\right\}+\sqrt{n h_{n}^{d}} \mathcal{D} \mathcal{I}_{\alpha} .
\end{aligned}
$$

Under the null hypothesis $H_{0}^{e q}$, we have: $\mathcal{D} \mathcal{I}_{\alpha}=0$

$$
\begin{aligned}
\widehat{\mathcal{D I}}_{n} & =\sqrt{n h_{n}^{d}}\left\{\widehat{\mathcal{D}}_{1}-\mathcal{D}_{1}\right\}-\sqrt{n h_{n}^{d}}\left\{\widehat{\mathcal{D}}_{2}-\mathcal{D}_{2}\right\} \\
& =\sqrt{n h_{n}^{d}} \psi^{\prime}\left(\int_{\mathbb{R}^{d}} \phi\left(\frac{f(x)}{f_{1}(x)}\right) f_{1}(x) d x\right)
\end{aligned}
$$


GENERALIZED DIVERGENCE CRITERIA FOR ...

95

$$
\begin{aligned}
& \times\left\{\int_{\mathbb{R}^{d}} \phi\left(\frac{\widehat{f}_{n h_{n}}(x)}{f_{1}(x)}\right) f_{1}(x) d x-\int_{\mathbb{R}^{d}} \phi\left(\frac{f(x)}{f_{1}(x)}\right) f_{1}(x) d x\right\}+o_{p}(1) \\
& -\sqrt{n h_{n}^{d}}{\psi^{\prime}}\left(\int_{\mathbb{R}^{d}} \phi\left(\frac{f(x)}{f_{2}(x)}\right) f_{2}(x) d x\right) \\
& \times\left\{\int_{\mathbb{R}^{d}} \phi\left(\frac{\widehat{f}_{n h_{n}}(x)}{f_{2}(x)}\right) f_{2}(x) d x-\int_{\mathbb{R}^{d}} \phi\left(\frac{f(x)}{f_{2}(x)}\right) f_{1}(x) d x\right\}-o_{p}(1) \\
& =\sqrt{n h_{n}^{d}} \psi^{\prime}\left(\int_{\mathbb{R}^{d}} \phi\left(\frac{f(x)}{f_{1}(x)}\right) f_{1}(x) d x\right) \\
& \times\left\{\int_{\mathbb{R}^{d}}\left(\widehat{f}_{n h_{n}}(x)-f(x)\right) \phi^{\prime}\left(\frac{f(x)}{f_{1}(x)}\right) d x+\int_{\mathbb{R}^{d}} o\left(\frac{\widehat{f}}{f_{1}}-\frac{f}{f_{1}}\right) f_{1}(x) d x\right\} \\
& +o_{p}(1)-\sqrt{n h_{n}^{d}} \psi^{\prime}\left(\int_{\mathbb{R}^{d}} \phi\left(\frac{f(x)}{f_{2}(x)}\right) f_{2}(x) d x\right) \\
& \times\left\{\int_{\mathbb{R}^{d}}\left(\widehat{f}_{n h_{n}}(x)-f(x)\right) \phi^{\prime}\left(\frac{f(x)}{f_{2}(x)}\right) d x+\int_{\mathbb{R}^{d}} o\left(\frac{\widehat{f}_{n h_{n}}(x)}{f_{2}(x)}-\frac{f(x)}{f_{2}(x)}\right) f_{2}(x) d x\right\}-o_{p}(1) \\
& =\psi^{\prime}\left(\int_{\mathbb{R}^{d}} \phi\left(\frac{f(x)}{f_{1}}\right) f_{1}(x) d x\right) \\
& \times\left\{\int_{\mathbb{R}^{d}} \sqrt{n h_{n}^{d}}\left(\hat{f}_{n h_{n}}(x)-f(x)\right) \phi^{\prime}\left(\frac{f(x)}{f_{1}(x)}\right) d x+\int_{\mathbb{R}^{d}} o_{p}(1) f_{1}(x) d x\right\} \\
& -\psi^{\prime}\left(\int_{\mathbb{R}^{d}} \phi\left(\frac{f(x)}{f_{2}(x)}\right) f_{2}(x) d x\right) \\
& \times\left\{\int_{\mathbb{R}^{d}} \sqrt{n h_{n}^{d}}\left(\widehat{f}_{n h_{n}}(x)-f(x)\right) \phi^{\prime}\left(\frac{f(x)}{f_{2}(x)}\right) d x+\int_{\mathbb{R}^{d}} o_{p}(1) f_{2}(x) d x\right\} \\
& +o_{p}(1)
\end{aligned}
$$




$$
\begin{aligned}
= & \int_{\mathbb{R}^{d}}\left\{\psi^{\prime}\left(\int_{\mathbb{R}^{d}} \phi\left(\frac{f(x)}{f_{1}(x)}\right) f_{1}(x) d x\right) \phi^{\prime}\left(\frac{f(x)}{f_{1}(x)}\right)+o_{p}(1) f_{1}(x)\right. \\
& -\psi^{\prime}\left(\int_{\mathbb{R}^{d}} \phi\left(\frac{f(x)}{f_{2}(x)}\right) f_{2}(x) d x\right) \phi^{\prime}\left(\frac{f(x)}{f_{2}(x)}\right) \\
& \left.-o_{p}(1) f_{2}(x)\right\} \times \sqrt{n h_{n}^{d}}\left(\widehat{f}_{n h_{n}}(x)-f(x)\right) d x+o_{p}(1) .
\end{aligned}
$$

Therefore

$$
\sqrt{n h_{n}^{d}} \widehat{\mathcal{D I}}_{n} \rightarrow \mathcal{N}\left(0, \Gamma^{2}\right)
$$

where

$$
\begin{aligned}
\Gamma^{2}=\left\{\int _ { \mathbb { R } ^ { d } } \left[\psi^{\prime}\left(\int_{\mathbb{R}^{d}} \phi\left(\frac{f(x)}{f_{1}(x)}\right) f_{1}(x) d x\right) \phi^{\prime}\left(\frac{f(x)}{f_{1}(x)}\right)\right.\right. \\
\left.\left.-\psi^{\prime}\left(\int_{\mathbb{R}^{d}} \phi\left(\frac{f(x)}{f_{2}(x)}\right) f_{2}(x) d x\right) \phi^{\prime}\left(\frac{f(x)}{f_{2}(x)}\right)\right] \sigma(x) d x\right\}^{2} .
\end{aligned}
$$

Note that in the case of the $\alpha$-divergence the asymptotic variance $\Gamma^{2}$ is

$$
\begin{aligned}
\Gamma^{2}:= & \Gamma^{2}(\alpha) \\
= & \left\{\int _ { \mathbb { R } ^ { d } } \left[\psi^{\prime}\left(\int_{\mathbb{R}^{d}} \phi\left(\frac{f(x)}{f_{1}(x)}\right) f_{1}(x) d x\right) \phi^{\prime}\left(\frac{f(x)}{f_{1}(x)}\right)\right.\right. \\
& \left.\left.-\psi^{\prime}\left(\int_{\mathbb{R}^{d}} \phi\left(\frac{f(x)}{f_{2}(x)}\right) f_{2}(x) d x\right) \phi^{\prime}\left(\frac{f(x)}{f_{2}(x)}\right)\right] \sigma(x) d x\right\}^{2},
\end{aligned}
$$

with $\psi(x)=x$ and $\phi(x)=\frac{1}{\alpha(\alpha-1)}\left(x^{\alpha}-\alpha(x-1)-1\right)$

$$
\Gamma^{2}(\alpha)=\left\{\frac{1}{\alpha-1} \int_{\mathbb{R}^{d}}\left[\left(\frac{f(x)}{f_{1}(x)}\right)^{\alpha-1}-\left(\frac{f(x)}{f_{2}(x)}\right)^{\alpha-1}\right] \sqrt{f(x)} d x \int_{\mathbb{R}^{d}} K^{2}(z) d z\right\}^{2} .
$$


In the special case where $\alpha=1 / 2$, this asymptotic variance does not depend to the unknown density $f$ and it is expressed by:

$$
\Gamma^{2}(1 / 2)=\left\{2 \int_{\mathbb{R}^{d}}\left(\sqrt{f_{1}(x)}-\sqrt{f_{2}(x)}\right) d x \int_{\mathbb{R}^{d}} K^{2}(z) d z\right\}^{2} .
$$

But the case $\alpha \neq 1 / 2, \Gamma^{2}(\alpha)$ is unknown because it depends on $f$ which is also unknown. In practice, one way solve this problem is to substitute $f$ with its consistency kernel estimator $\widehat{f}_{n h_{n}}$ and to plug it in $\Gamma^{2}(\alpha)$.

\section{Computational Results}

\subsection{Example}

To illustrate the model procedure discussed in the preceding section, we consider an example.

We consider various sets of experiments in which data are generated from the mixture of a Normal $\mathcal{N}(0,1)$ and Normal $\mathcal{N}(0,2.5)$ distributions. Hence, the DGP (Data Generating Process) is generated from $m(\pi)$ with the density

$$
m(\pi)=\pi \mathcal{N}(0,1)+(1-\pi) \mathcal{N}(0,2.5),
$$

where $\pi(\pi \in[0,1])$ is specific value to each set of experiments. In each set of experiments, severals random sample are drawn from this mixture of distributions. The sample size varies from 100 to 2000, and for each sample size the number of replication is 1000 . We choose value of the parameter $\alpha=0.5$, that corresponds to the Hellinger distance (this choice provided to the known asymptotic variance). The aim is to compare the distance between true density and the density $\mathcal{N}(0,1)$, and the distance between the true density and the density $\mathcal{N}(0,2.5)$. 
We choose different values of $\pi$ which are $0.00,0.25,0.43,0.75,1.00$. Although our proposed model selection procedure does not require that the data generating process belong to either of the competing models, we consider the two limiting cases $\pi=1.00$ and $\pi=0.00$ for they correspond to the correctly specified cases. To investigate the case where both competing models are misspecified but not at equal distance from the DGP, we consider the case $\pi=0.25, \pi=0.75$, and $\pi=0.43$. Second case is interpreted similarly as a $\mathcal{N}(0,2.5)$ slightly contaminated by a $\mathcal{N}(0,1)$ distribution. The former case correspond to a DGP which is $\mathcal{N}(0,1)$ but slightly contaminated by a $\mathcal{N}(0,2.5)$ distribution. In the last case, $\pi=0.43$ is the value for which the $\widehat{\mathcal{D}}_{\alpha}\left(\widehat{f}_{n}, f_{1}\right)$ and the $\widehat{\mathcal{D}}_{\alpha}\left(\widehat{f}_{n}, f_{2}\right)$ family are approximately at equal distance to the mixture $m(\pi)$ according to the $\alpha$-divergence with the above cells. Thus, this series of experiments approximates the null hypothesis of our proposed model selection test $\widehat{\mathcal{D I}}_{\alpha}$. The results of our different sets of experiments are presented in Tables 1-5.

Table 1. $D G P=\mathcal{N}(0,1)$

\begin{tabular}{lcccccccc}
\hline$n$ & & 20 & 100 & 300 & 500 & 1000 & 1500 & 2000 \\
\hline$\widehat{\mathcal{D}}_{1}$ & & -0.05 & 0.007 & -0.002 & 0.016 & -0.004 & 0.012 & 0.006 \\
$\widehat{\mathcal{D}}_{2}$ & & 0.16 & 0.12 & 0.14 & 0.16 & 0.14 & 0.14 & 0.14 \\
$\widehat{\mathcal{D I}}_{\alpha}$ & & -0.21 & -0.11 & -0.15 & -0.14 & -0.146 & -0.12 & -0.14 \\
& & & & & & & & \\
& Correct & $8.4 \%$ & $8 \%$ & $26.4 \%$ & $57.8 \%$ & $95.6 \%$ & $100 \%$ & $100 \%$ \\
& Indecisive & $91.6 \%$ & $92 \%$ & $73.6 \%$ & $42.2 \%$ & $4.4 \%$ & $0 \%$ & $0 \%$ \\
& Incorrect & $0 \%$ & $0 \%$ & $0 \%$ & $0 \%$ & $0 \%$ & $0 \%$ & $0 \%$ \\
\hline
\end{tabular}


Table 2. $D G P=\mathcal{N}(0,2.5)$

\begin{tabular}{|c|c|c|c|c|c|c|c|c|}
\hline$n$ & & 20 & 100 & 300 & 500 & 1000 & 1500 & 2000 \\
\hline$\widehat{\mathcal{D}}_{1}$ & & 0.26 & 0.14 & 0.22 & 0.28 & 0.23 & 0.24 & 0.24 \\
\hline$\widehat{\mathcal{D}}_{2}$ & & -0.039 & -0.016 & -0.008 & -0.006 & -0.004 & -0.002 & -0.001 \\
\hline \multirow[t]{4}{*}{$\widehat{\mathcal{D I}}_{\alpha}$} & & 0.30 & 0.16 & 0.23 & 0.29 & 0.23 & 0.24 & 0.24 \\
\hline & Correct & $30.8 \%$ & $68.4 \%$ & $94.2 \%$ & $99 \%$ & $100 \%$ & $100 \%$ & $100 \%$ \\
\hline & Indecisive & $69 \%$ & $31.6 \%$ & $5.6 \%$ & $1 \%$ & $0 \%$ & $0 \%$ & $0 \%$ \\
\hline & Incorrect & $0.2 \%$ & $0 \%$ & $0.2 \%$ & $0 \%$ & $0 \%$ & $0 \%$ & $0 \%$ \\
\hline
\end{tabular}

Table 3. $D G P=.75 * \mathcal{N}(0,1)+.25 * \mathcal{N}(0,2.5)$

\begin{tabular}{|c|c|c|c|c|c|c|c|c|}
\hline$n$ & & 20 & 100 & 300 & 500 & 1000 & 1500 & 2000 \\
\hline$\widehat{\mathcal{D}}_{1}$ & & -0.014 & 0.015 & -0.001 & 0.01 & -0.002 & 0.01 & 0.01 \\
\hline$\widehat{\mathcal{D}}_{2}$ & & 0.19 & 0.19 & 0.16 & 0.13 & 0.13 & 0.11 & 0.12 \\
\hline \multirow[t]{4}{*}{$\widehat{\mathcal{D I}}_{\alpha}$} & & -0.21 & -0.17 & -0.16 & -0.12 & -0.13 & -0.1 & -0.11 \\
\hline & $\mathcal{N}(0,1)$ & $1.6 \%$ & $5.4 \%$ & $34.4 \%$ & $67.4 \%$ & $99 \%$ & $100 \%$ & $100 \%$ \\
\hline & Indecisive & $98.4 \%$ & $94.6 \%$ & $64.4 \%$ & $32.6 \%$ & $1 \%$ & $0 \%$ & $0 \%$ \\
\hline & $\mathcal{N}(0,2.5)$ & $0 \%$ & $0 \%$ & $0 \%$ & $0 \%$ & $0 \%$ & $0 \%$ & $0 \%$ \\
\hline
\end{tabular}

Table 4. $D G P=.43 * \mathcal{N}(0,1)+.57 * \mathcal{N}(0,2.5)$

\begin{tabular}{|c|c|c|c|c|c|c|c|c|}
\hline$n$ & & 20 & 100 & 300 & 500 & 1000 & 1500 & 2000 \\
\hline$\widehat{\mathcal{D}}_{1}$ & & 0.1 & 0.05 & 0.04 & 0.05 & 0.04 & 0.053 & 0.057 \\
\hline$\widehat{\mathcal{D}}_{2}$ & & 0.08 & 0.02 & 0.06 & 0.04 & 0.05 & 0.056 & 0.058 \\
\hline \multirow[t]{4}{*}{$\widehat{\mathcal{D I}}_{\alpha}$} & & 0.02 & 0.03 & -0.02 & 0.01 & -0.01 & -0.002 & -0.01 \\
\hline & $\mathcal{N}(0,1)$ & $1.4 \%$ & $0.2 \%$ & $0.2 \%$ & $0 \%$ & $0 \%$ & $0 \%$ & $0 \%$ \\
\hline & Indecisive & $98.4 \%$ & $99.8 \%$ & $99.8 \%$ & $100 \%$ & $100 \%$ & $100 \%$ & $100 \%$ \\
\hline & $\mathcal{N}(0,2.5)$ & $0.2 \%$ & $0 \%$ & $0 \%$ & $0 \%$ & $0 \%$ & $0 \%$ & $0 \%$ \\
\hline
\end{tabular}


Table 5. $D G P=.25 * \mathcal{N}(0,1)+.75 * \mathcal{N}(0,2.5)$

\begin{tabular}{|c|c|c|c|c|c|c|c|c|}
\hline$n$ & & 20 & 100 & 300 & 500 & 1000 & 1500 & 2000 \\
\hline$\widehat{\mathcal{D}}_{1}$ & & 0.69 & 0.83 & 1.006 & 0.86 & 1.08 & 1.04 & 0.99 \\
\hline$\widehat{\mathcal{D}}_{2}$ & & -0.024 & 0.039 & 0.02 & 0.06 & 0.05 & 0.046 & 0.06 \\
\hline \multirow[t]{4}{*}{$\widehat{\mathcal{D I}}_{\alpha}$} & & 0.67 & 0.79 & 1.04 & 0.8 & 1.03 & 0.99 & 0.92 \\
\hline & $\mathcal{N}(0,1)$ & $0.6 \%$ & $0 \%$ & $0 \%$ & $0 \%$ & $0 \%$ & $0 \%$ & $0.1 \%$ \\
\hline & Indecisive & $21 \%$ & $17 \%$ & $0.4 \%$ & $0.2 \%$ & $0.2 \%$ & $0.2 \%$ & $0.1 \%$ \\
\hline & $\mathcal{N}(0,2.5)$ & $78.4 \%$ & $83 \%$ & $99.6 \%$ & $99.8 \%$ & $99.8 \%$ & $99.8 \%$ & $99.9 \%$ \\
\hline
\end{tabular}

Thus, this set of experiments corresponds approximately to the null hypothesis of our proposed model selection test $\widehat{\mathcal{D I}}$. The results of our different sets of experiments are presented in Tables 1-5. The first half of each table gives the distance between the true density $f$ and $f_{1}$ sample take density model $1 \mathcal{D}_{1}$, the distance between $f$ and $f_{2}$ model $2 \mathcal{D}_{2}$ and the difference between the two distance. The second half of each table gives in percentage the number of times our proposed model selection procedure based on $\widehat{\mathcal{D I}}$ favors the model 1, the model 2, and indecisive. The tests are conducted at 5\% nominal significance level. In the first two sets of experiments $(\pi=0.00$ and $\pi=1.00)$ where one model is correctly specified, we use the labels "correct, incorrect" and "indecisive" when a choice is made. The first halves of Tables 1-5 confirm our asymptotic results. In Table 4, we observed a high percentage of bad decisions. This is because both models are now specified incorrectly. In contrast, turning to the second halves of the Tables 1 and 2, we first note that the percentage of correct choices using $\mathcal{D I}$ statistic steadily increases and ultimately converges to $100 \%$. The preceding comments for the second halves of Tables 1 and 2 also apply to the second halves of Tables 3 and 5 .

In Figures 1, 3, 5, 7, and 9, we plot the histograms of data sets and overlay the curves for $\mathcal{N}(0,1)$ and $\mathcal{N}(0,2.5)$ distributions. When the DGP is correctly specified Figure 1 , the $\mathcal{N}(0,1)$ distribution has reasonable chance of being distinguished from $\mathcal{N}(0,1)$ distribution. 


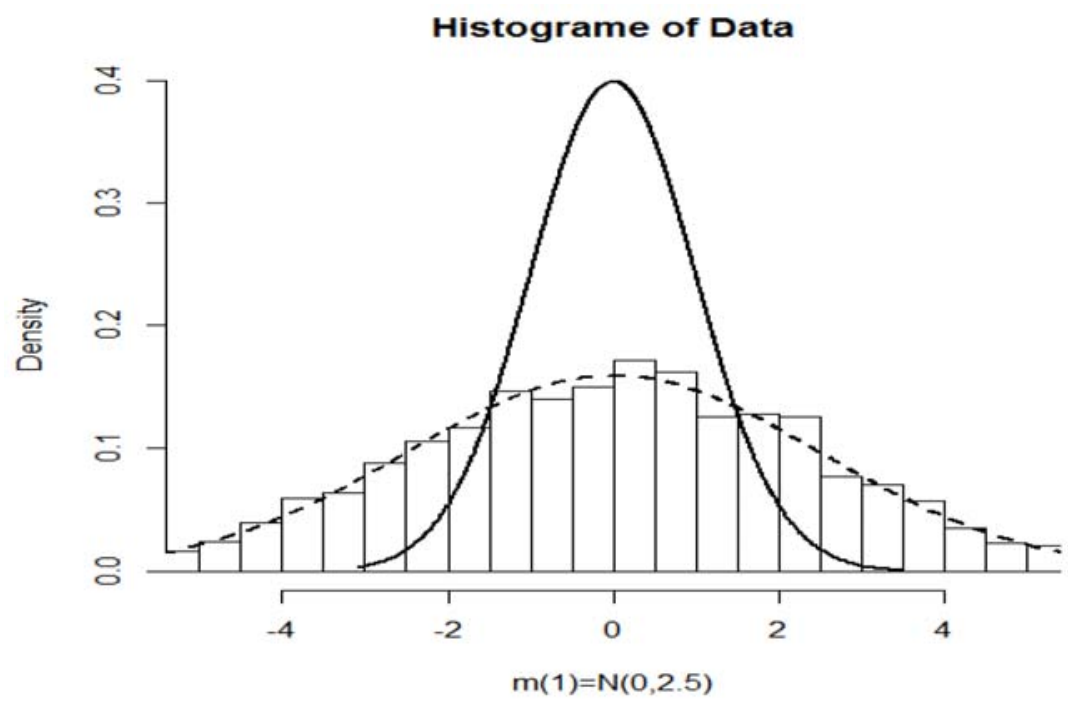

Figure 1. Histogram of $(D G P=\mathcal{N}(0,1))$.

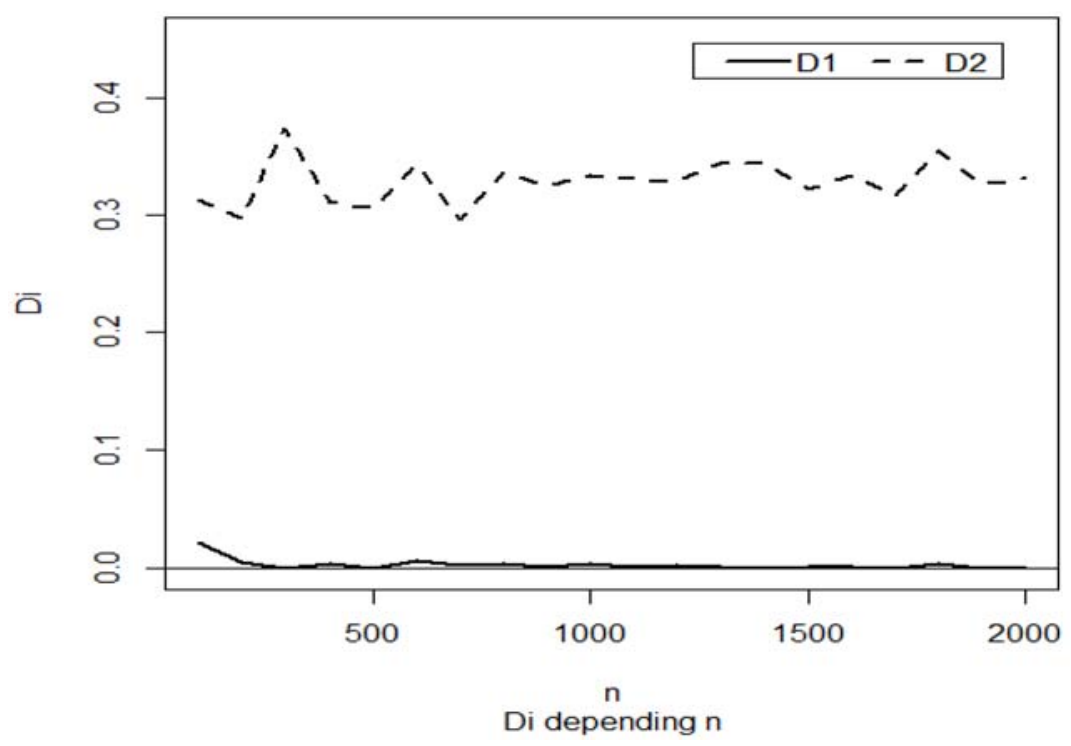

Figure 2. $\widehat{\mathcal{D}}_{1}$ and $\widehat{\mathcal{D}}_{2}$ depending $n$. 


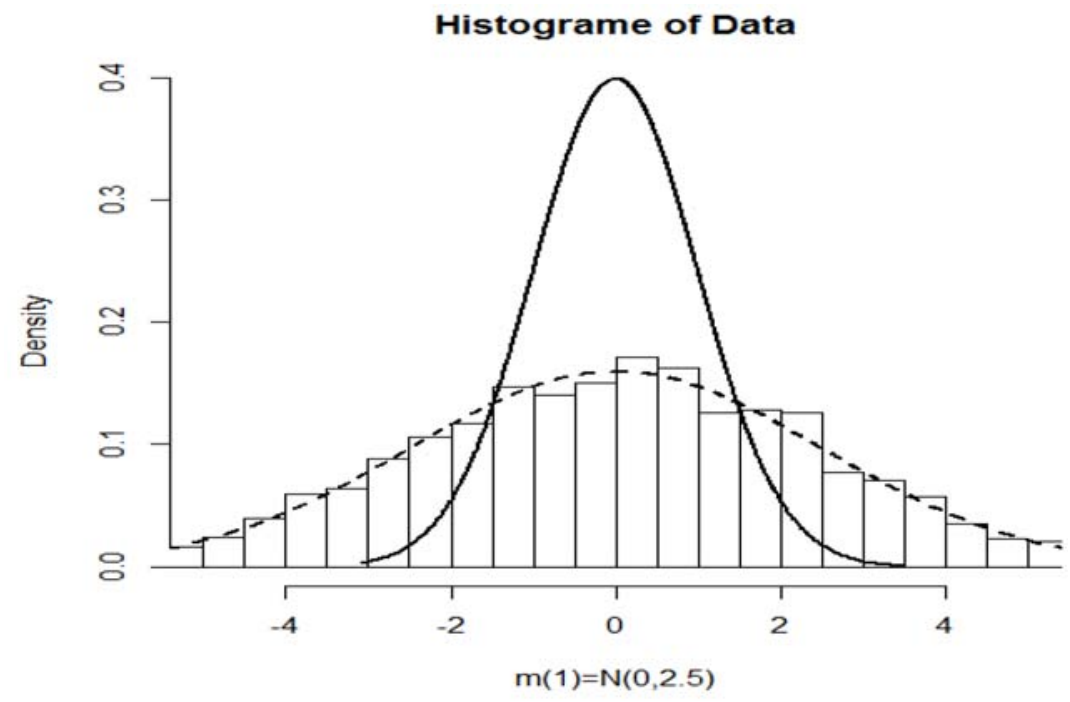

Figure 3. Histogram of $(D G P=\mathcal{N}(0,2.5))$.

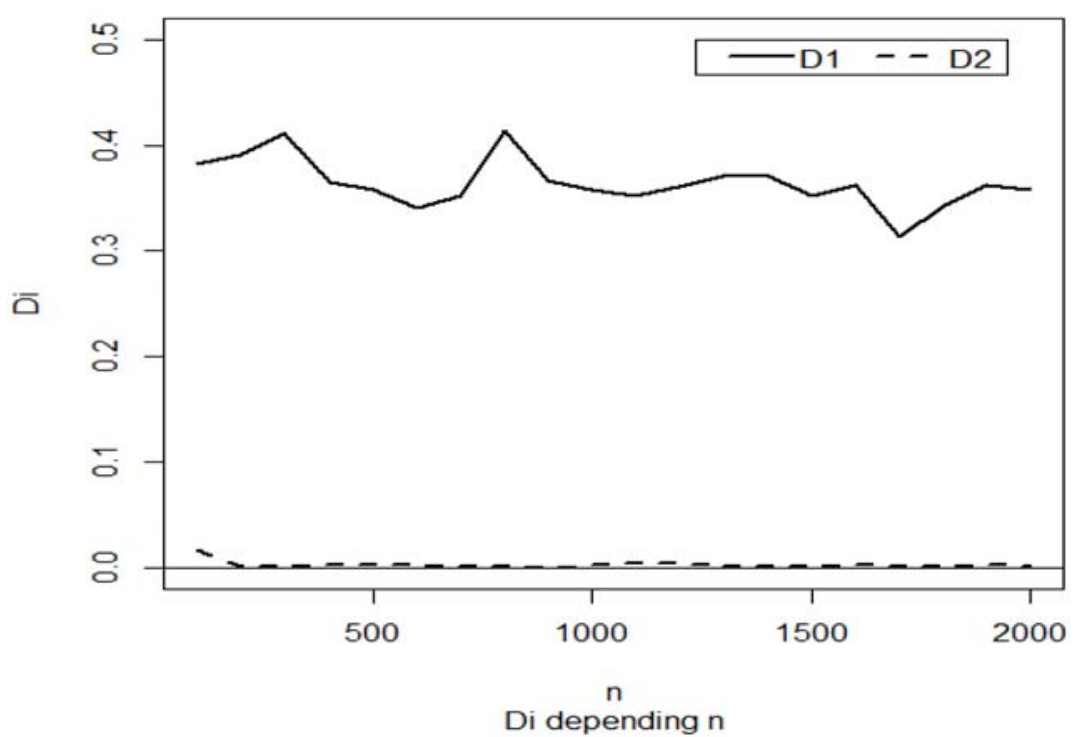

Figure 4. $\widehat{\mathcal{D}}_{1}$ and $\widehat{\mathcal{D}}_{2}$ depending $n$. 


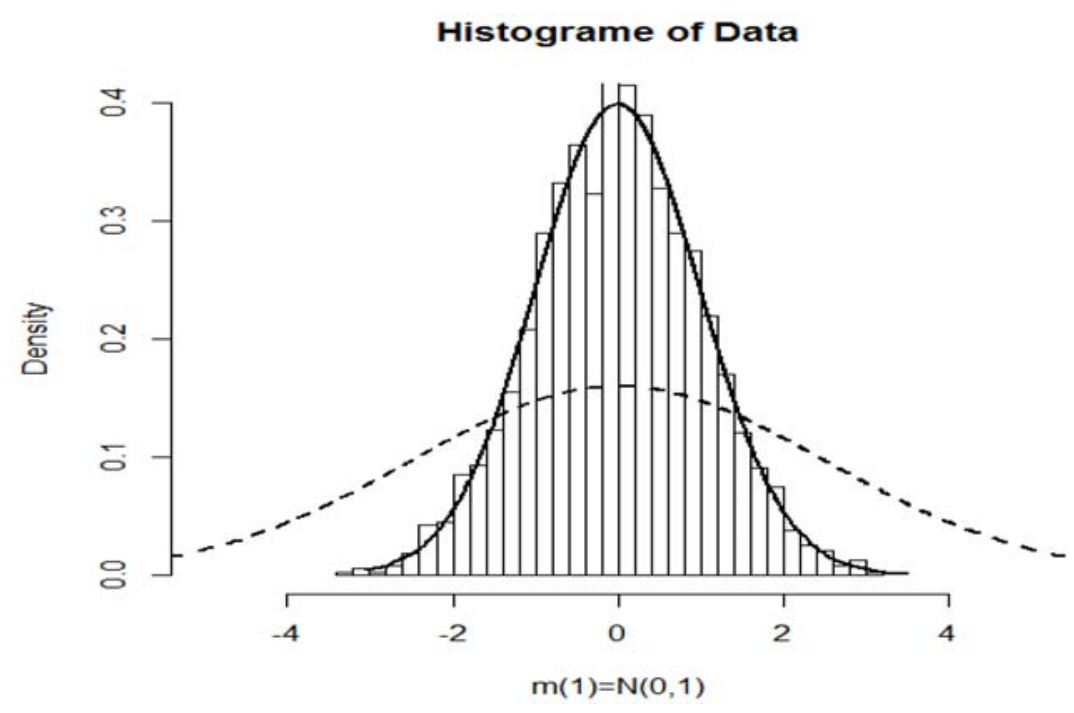

Figure 5. Histogram of $(D G P=.75 * \mathcal{N}(0,1)+.25 * \mathcal{N}(0,2.5))$.

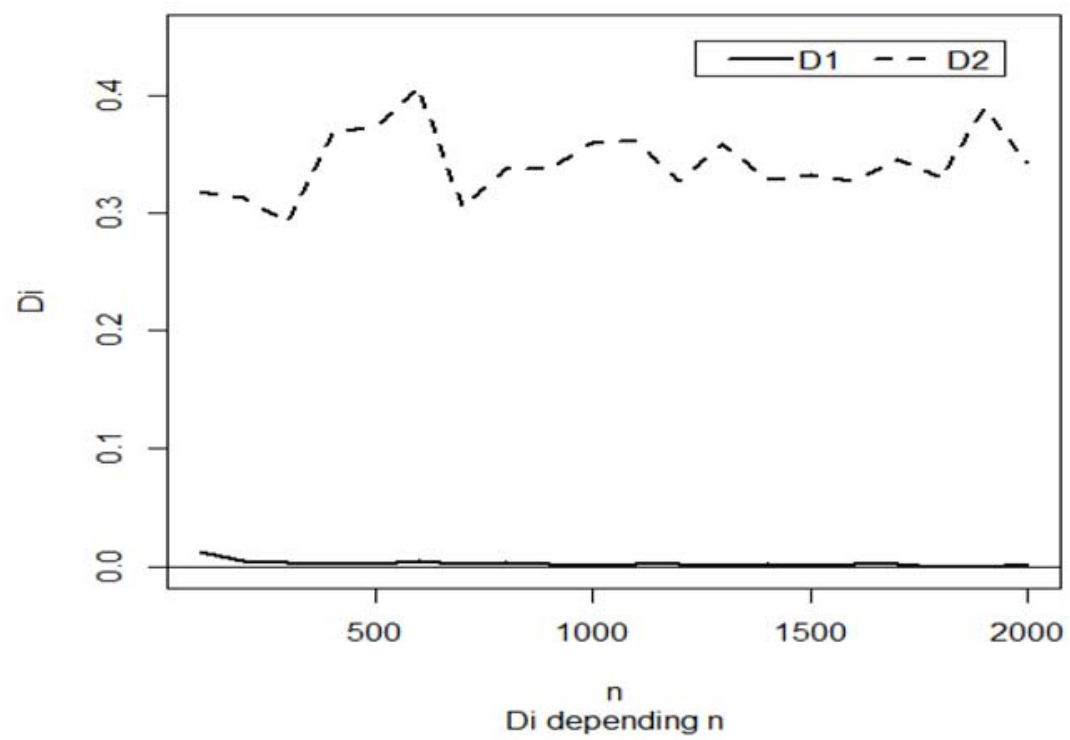

Figure 6. $\widehat{\mathcal{D}}_{1}$ and $\widehat{\mathcal{D}}_{2}$ depending $n$. 


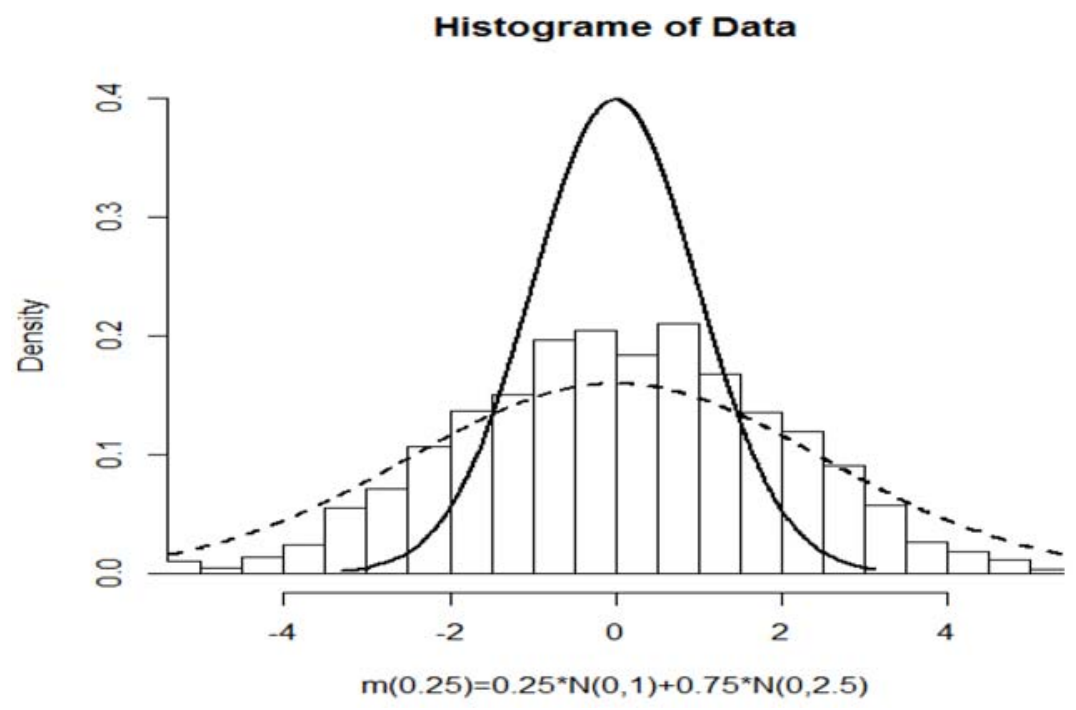

Figure 7. Coparaison barplot of Di depending $n(D G P=.25 * \mathcal{N}(0,1)+$ $.75 * \mathcal{N}(0,2.5))$.

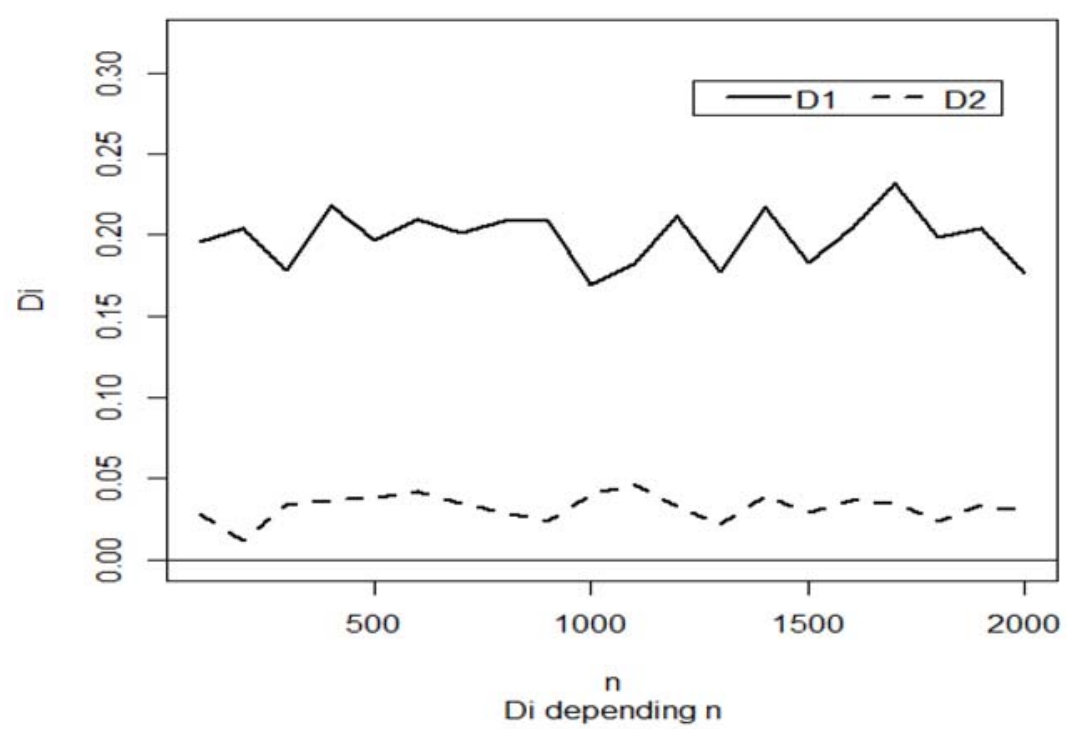

Figure 8. $\widehat{\mathcal{D}}_{1}$ and $\widehat{\mathcal{D}}_{2}$ depending $n$. 


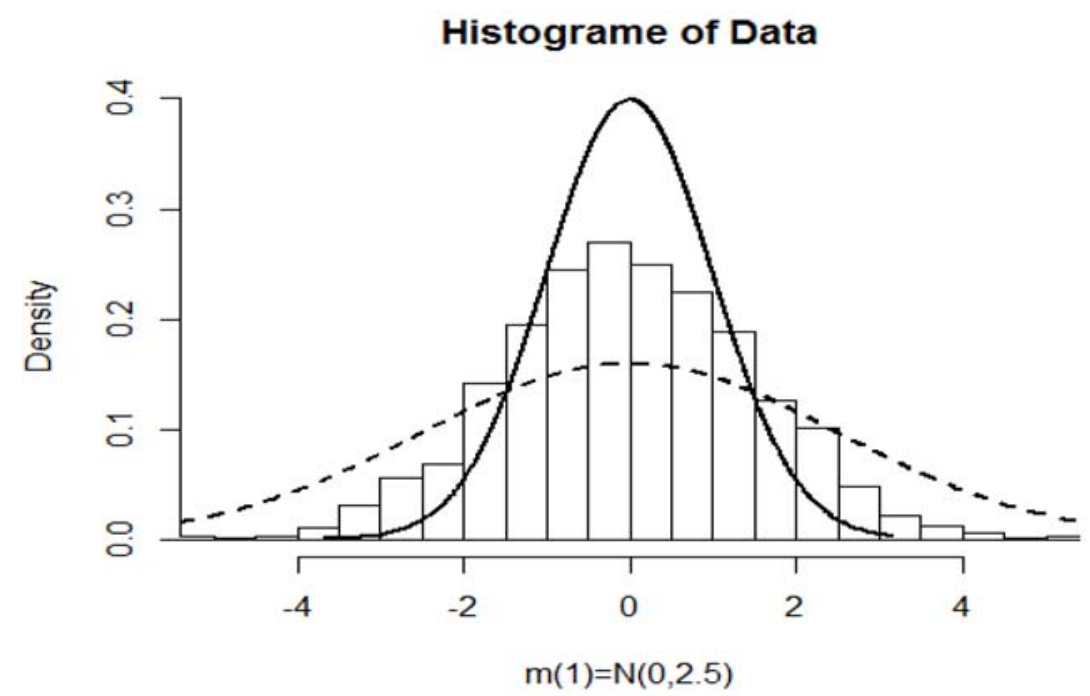

Figure 9. Coparaison barplot of Di depending $n(D G P=.43 * \mathcal{N}(0,1)+$ $.57 * \mathcal{N}(0,2.5))$.

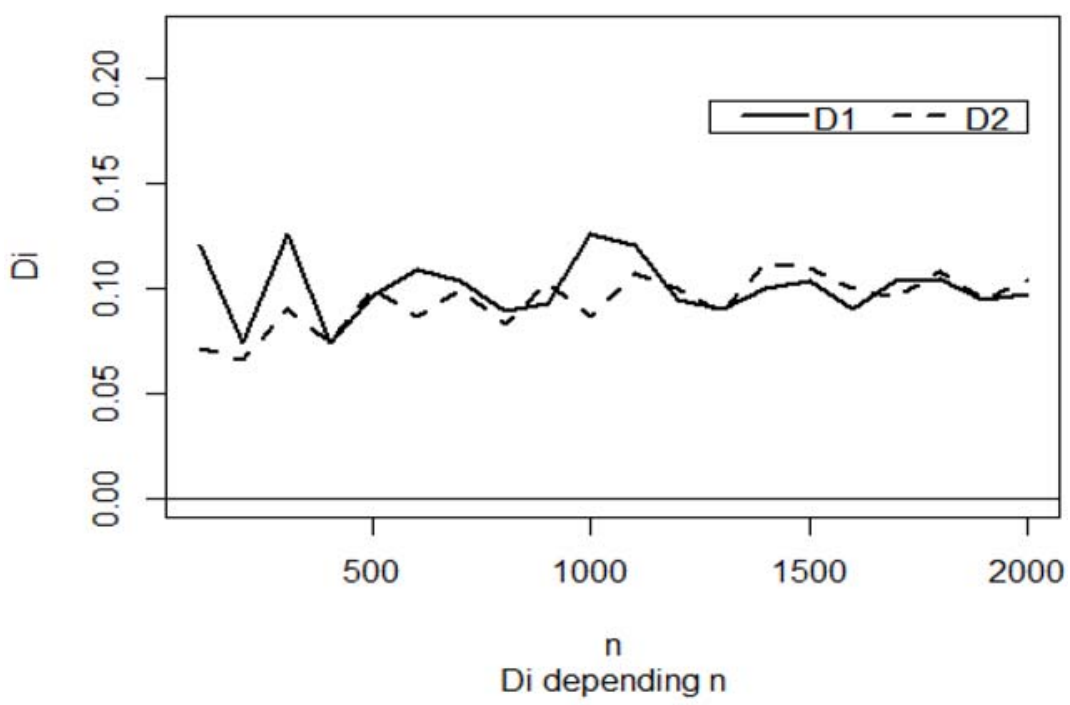

Figure 10. $\widehat{\mathcal{D}}_{1}$ and $\widehat{\mathcal{D}}_{2}$ depending $n$. 


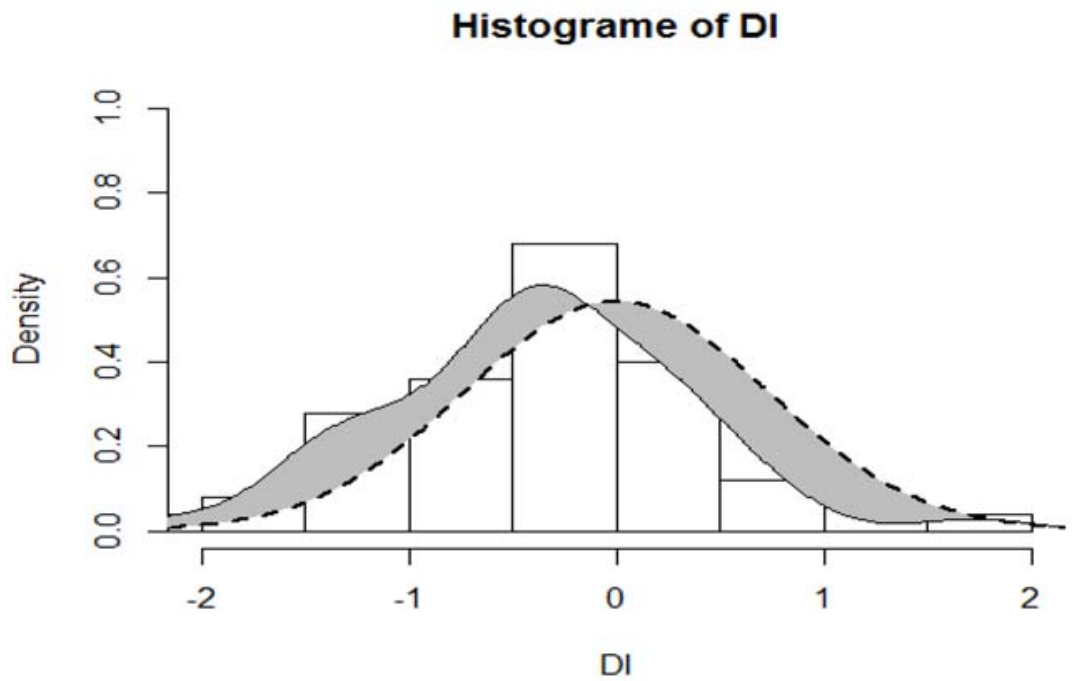

Figure 11. Coparaison of density depending $n$ density data (continuous curve), density $\mathcal{N}(0, \Gamma)$ (dashed curve).

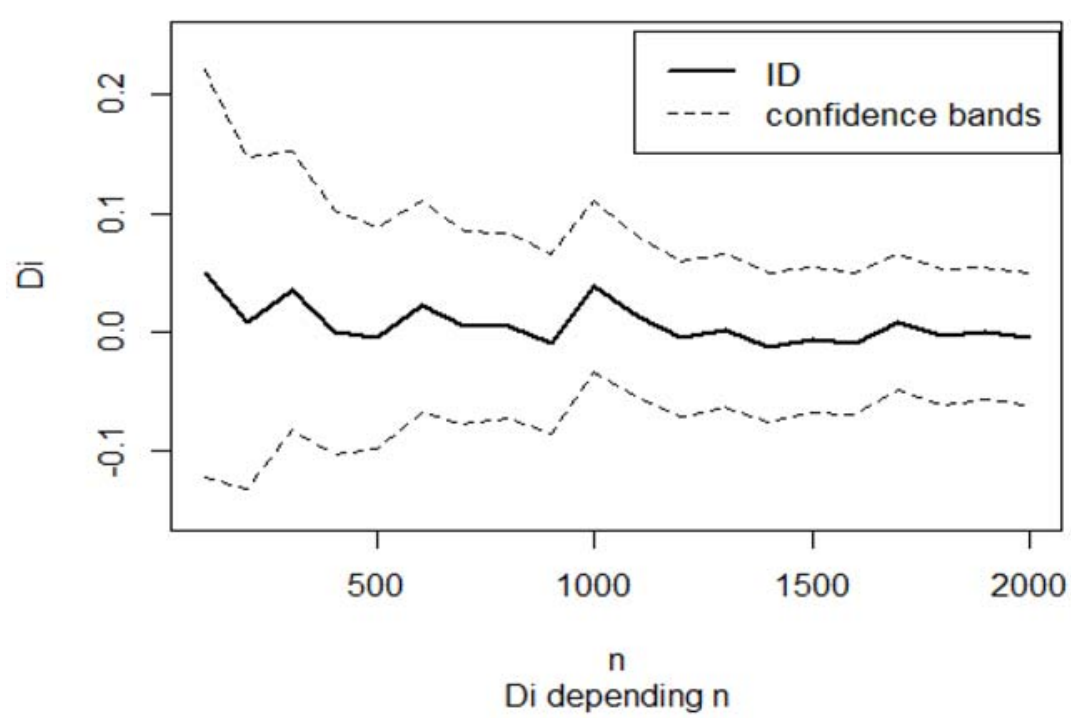

Figure 12. $\widehat{\mathcal{I D}}$ depending $n$ and its confidence band at the levels $95 \%$. 
Similarly, in Figure 3 , as can be seen, the $\mathcal{N}(0,2.5)$ distribution closely approximates the data sets. In Figures 5 and 7, two distributions are close but the $\mathcal{N}(0,1)$ (Figure 5) and the $\mathcal{N}(0,2.5)$ distributions (Figure 7) does appear to be much closer to the data sets. When $\pi=0.43$, the distribution for both (Figure 9) $\mathcal{N}(0,1)$ distribution and $\mathcal{N}(0,2.5)$ distribution are similar.

As expected, our statistic divergence $\sqrt{n h_{n}^{d}} \widehat{\mathcal{D I}}_{\alpha}$ diverges to $-\infty$ (Figures 2 and 6) and to $+\infty$ (Figures 4 and 8) more rapidly symmetrical about the axis that passes through the mode of data distribution. This follows from the fact that these two distributions are equidistant from the DGP and would be difficult to distinguish from data in practice.

Figure 10 allows a comparison with the asymptotic $\mathcal{N}(0, \Gamma)$ approximation under our null hypothesis of equivalence. Figure 11, hence the density indicator $\widehat{\mathcal{D I}}_{\alpha}$ is very closer to the $\mathcal{N}(0, \Gamma)$.

\section{Conclusion}

We learned a new nonparametric estimation for the divergence measure, and has been applied to the problems of model selection. Under certain conditions, we have shown the consistency of these estimators and how they can be applied to estimate the distance between a known density and an unknown density other than estimated by the kernel method. Our tests are based on testing whether the competing models are equally close to the true distribution against the alternative hypotheses that one model is closer than the other where closeness of a model is measured according to the discrepancy implicit in the divergence type statistics used. We have also demonstrated their effectiveness by using numerical experiments. 


\section{References}

[1] H. Akaike, An approximation to the density function, Ann. Inst. Statist. Math. Tokyo 6 (1954), 127-132.

[2] S. Bouzebda and I. Elhattab, Uniform-in-bandwidth consistency for kernel-type estimators of Shannon's entropy, Electronic Journal of Statistics 5 (2011), 440-459.

[3] D. Bosq and J. P. Lecoutre, Théorie de l'estimation fonctionnelle, Économie et Statistiques Avancées, Economica, Paris, 1987.

[4] J. Burbea and C. R. Rao, On the convexity of divergence measures based on entropy functions, IEEE Trans. Inform. Theory 28 (1982a), 489-495.

[5] J. Burbea and C. R. Rao, On the convexity of higher order Jensen differences based on entropy functions, IEEE Trans. Inform. Theory 28 (1982b), 961-963.

[6] J. Burbea and C. R. Rao, Entropy differential metric distance and divergence measures in probability spaces: A unified approach, J. Multivariate Anal. 12 (1982c), 575-596.

[7] I. Csiszr, Information-type measures of differences of probability distributions and indirect observations, Studia Sci. Math. Hungarica 2 (1967), 299-318.

[8] P. Deheuvels, Uniform limit laws for kernel density estimators on possibly unbounded intervals, Stat. Ind. Technol. (2000), 477-492.

[9] P. Deheuvels and J. Einmahl, On the strong limiting behavior of local functionals of empirical processes based upon censored data, Ann. Prob. 24 (1996), 504-525.

[10] P. Deheuvels and D. M. Mason, General asymptotic confidence bands based on kernel-type function estimators, Stat. Inference Stoch. Process 7 (2004), 225-277.

[11] L. Devroye and L. Gyorfi, Nonparametric Density Estimation, Wiley Series in Probability and Mathematical Statistics: Tracts on Probability and Statistics, John Wiley \& Sons Inc., New York, The L1 View, 1985.

[12] L. Devroye and G. Lugosi, Combinatorial Methods in density Estimation, Springer Series in Statistics, Springer-Verlag, New York, 2001.

[13] H. Dhaker, P. Ngom, P. Mendy and E. Deme, Uniform-in-bandwidth consistency for nonparametric estimation of divergence measures, arXiv:1406.6017.

[14] U. Einmahl and D. M. Mason, An empirical process approach to the uniform consistency of kernel-type function estimators, J. Theoret. Probab. 13 (2000), 1-37.

[15] H. Jeffreys, An invariant form for the prior probability in estimation problems, Proceedings of the Royal Society of London, Series A, Mathematical and Physical Sciences 186(1007) (1946), 453-461.

[16] S. Kullback and R. A. Leibler, On information and sufficiency, The Annals of Mathematical Statistics 22 (1951), 79-86.

[17] A. Lynda and F. Hocine, On the stability of the unit root test, Journal Afrika Statistika 5 (2010), 228-237. 
[18] J. Marriott and P. Newbold, Bayesian comparison of ARIMA and stationary ARMA models, International Statistical Review 3 (1998), 323-336.

[19] M. L. Menendez, D. Morales, L. Pardo and I. Vajda, Divergence based estimation and testing of statistical models of classification, J. Multivariate Anal. 54 (1995), 329-354.

[20] D. B. Owen, Statistical Inference Based on Divergence Measures, Taylor \& Francis Group, LLC, 2006.

[21] E. Parzen, On estimation of a probability density function and mode, Ann. Math. Statist. 33 (1962), 1065-1076.

[22] B. Póczos and J. Schneider, On the estimation of alpha-divergences, CMU, Auton Lab Technical Report.

http://www.cs.cmu.edu/bapoczos/articles/poczos11alphaTR.pdf

[23] B. L. S. Prakasa Rao, Nonparametric Functional Estimation, Probability and Mathematical Statistics, Academic Press Inc. [Harcourt Brace Jovanovich Publishers], New York, 1983.

[24] A. Rényi, On measures of entropy and information, In Fourth Berkeley Symposium on Mathematical Statistics and Probability, 1961.

[25] B. D. Sharma and D. P. Mittal, New non-additive measures of relative information, Journ. Comb. Inf. Syst. Sci. 2 (1977), 122-132.

[26] M. Rosenblatt, Remarks on some nonparametric estimates of a density function, Ann. Math. Statist. 27 (1956), 832-837.

[27] I. J. Taneja, On Generalized Information Measures and their Applications, Chapter in: Advances in Electronics and Electon Physics, Editor P. W. Hawkes 76 (1989), 327-413. 